\title{
Living well with chronic illness: How social support, loneliness and psychological appraisals relate to well-being in a population-based European sample
}

Journal of Health Psychology I-14

(c) The Author(s) 2019

Article reuse guidelines: sagepub.com/journals-permissions DOI: $10.1177 / 1359105319883923$ journals.sagepub.com/home/hpq (SAGE

\author{
Rebecca Maguirel (D), Paul Hanly² \\ and Phil Maguire ${ }^{3}$
}

\begin{abstract}
Positive social and psychological appraisals may help maintain well-being in illness, but few studies have systematically investigated this in a European context. After controlling for sociodemographic, health and objective social factors, we employed multilevel regression modelling to establish if appraisals predict wellbeing in three illness groups $(n=10,577)$. In addition to health and financial status, positive appraisals were consistent predictors of well-being. Social appraisals had stronger associations with well-being than extent of social contact. Optimism and resilience appeared more important in those limited by health than those who were not. Findings may inform interventions aimed at supporting people coping with illness.
\end{abstract}

\section{Keywords}

autonomy, chronic illness, loneliness, optimism, positive appraisals, psychological well-being, resilience, social support

\section{Introduction}

The prevalence of chronic illness is increasing internationally, with a considerable number of people now living with some form of disease. This is partly due to increases in life expectancy (Brennan et al., 2017), with an ageing population at greater risk of various conditions such as cancer, cardiovascular disease, diabetes and neurodegenerative disorders. While chronic illness is typically coupled with adverse effects and burdens (Busse and Blümel, 2010), many people cope well with their conditions and suffer few limitations in their daily lives (Delle Fave et al., 2017; Kim et al., 2016). Nevertheless, numerous studies have illustrated that those with chronic illness are at risk of lower psychological well-being than others in the general population (Steptoe et al., 2015). This may even be the case in people whose illness does not considerably impact on their daily activities.

\footnotetext{
'Department of Psychology, Maynooth University, Ireland ${ }^{2}$ School of Business, National College of Ireland, Ireland ${ }^{3}$ Department of Computer Science, Maynooth University, Ireland

\section{Corresponding author:}

Rebecca Maguire, Department of Psychology, Maynooth University, Maynooth, Co. Kildare, Ireland. Eircode: W23 F2H6.

Email: rebecca.maguire@mu.ie
} 
While a number of different definitions of well-being exist, this can broadly be defined as a subjective state predominated by positive feelings (McDowell, 2010). As enhanced psychological well-being is known to lead to greater health and longevity overall (Diener and Chan, 2011), understanding the factors that may help maintain well-being in chronic illness is important when considering how best to support patients in living well with their conditions. In this study, we focus on well-being as measured by the World Health Organization Mental WellBeing Index (WHO-5) (Topp et al., 2015), which is a widely used global rating scale for measuring subjective well-being.

Attempting to unpick the factors that enhance psychological well-being in any group is difficult. While a range of sociodemographic variables, such as age and gender, appear to associate with well-being (Bekker and van Mens-Verhulst, 2007; Ryff and Singer, 1996), studies have not always revealed consistent findings. Often, in the case of chronic illness, the strongest effect on well-being is one's functional health status, yet there are a range of other objective and subjective factors that can influence this, each of which may operate differently depending on the severity of daily living limitations (Eiser et al., 2001). For example, social support can influence well-being, with many studies demonstrating that those with stronger social support networks have better outcomes than those who do not (Reblin and Uchino, 2008). However, an even more important factor appears to be how one interprets this support. In other words, subjective appraisals, such as loneliness and feelings of social exclusion, can have an even stronger influence on well-being than the extent of social contact (Golden et al., 2009). Loneliness, in particular, has been shown to be very important in determining how people cope with illness, regardless of objective support. Some research has suggested that social isolation has a more detrimental impact on well-being than ill health itself (Lamu and Olsen, 2018).

Aside from perceptions of support, a range of other psychological appraisals may play an important role in maintaining well-being in those with chronic illness (De Ridder et al., 2008;
Maguire et al., 2018). For example, optimism has been demonstrated to be highly predictive of quality of life, with optimistic individuals more likely to report higher well-being (Karademas, 2006; Scheier et al., 2001) and better health outcomes (DuBois et al., 2015). Other studies, by contrast, have suggested that being overly optimistic may not be adaptive in certain diseases (Hurt et al., 2014) and that having a more realistic outlook may be more beneficial. Further important psychological appraisals include a person's level of resilience, autonomy and sense of purpose (Boyle et al., 2009; Dezutter et al., 2015; Edward, 2013). We have previously demonstrated how these appraisals are strongly associated with well-being among informal caregivers (Maguire et al., 2019). It is likely that positive appraisals may also play an important role in determining well-being in those suffering from chronic illness themselves.

The question of whether well-being in chronic illness can be separately predicted by different sociodemographic, social and psychological factors such as those outlined above has not been fully explored. While a number of studies have examined well-being in specific patient populations, few have investigated well-being in chronic illness more generally, or have attempted to unpick the relative importance of objective and subjective factors in those with different degrees of illness severity (with the exception of works such as Chakhssi et al. (2018) and Geerling et al. (2019)). In our study, using data from the European Quality of Life Surveys (EQLS), we wished to compare the well-being of three groups of people with a chronic condition who varied in the extent to which they were limited by their illness. We aimed to compare the associates of well-being in these groups with a focus on how subjective social and psychological appraisals, as distinct from reported social support, relate to well-being.

\section{Method}

\section{Participants}

The EQLS is conducted every 4 years by the European Foundation for the Improvement of 
Living and Working Conditions (Eurofound, 2018). This involves interviews with a random sample of adults in various European countries, including European Union (EU) member states (minimum sample size $=1000$ per country). Our study used data from the most recent survey, conducted in 2016-2017. Data were obtained via the UK Data Service, and ethical approval for conducting the analysis was granted from Maynooth University (reference: SRESC-2019-044). All participants in the EQLS gave informed consent.

There were 36,908 respondents in the EQLS. These came from 33 European countries, including all $28 \mathrm{EU}$ member states and an additional 5 candidate countries (Albania, FYR Macedonia, Montenegro, Serbia and Turkey). We were interested in respondents who reported having a chronic condition, specifically participants who responded 'yes' to the following question: 'Do you have any chronic (long-standing) physical or mental health problem, illness or disability? By chronic (long-standing) I mean illnesses or health problems which have lasted, or are expected to last, for 6 months or more'. A subsequent question asked participants whether they were limited in their daily activities by their chronic health condition. Possible responses were 'Yes, severely', 'Yes, to some extent' and 'No'. This gave rise to three different categories of people with chronic illness (no limitations, some limitations and severe limitations) which we focused on separately in our analyses.

\section{Measures}

Well-being. The WHO-5 was used to measure psychological well-being (Topp et al., 2015). Here, respondents rated the extent to which they felt a particular way in the previous 2 weeks on a scale of 1 (all of the time) to 5 (some of the time). There were five statements in all (e.g. 'I have felt cheerful and in good spirits') which were summed and standardised from 0 to 100 , with higher WHO-5 scores corresponding to higher levels of well-being. Overall, reliability for this scale was good in the sample (Cronbach's alpha $=0.891$ ).
Sociodemographic factors and health status. The EQLS contained numerous measures of sociodemographic characteristics, including gender, age, employment status (coded as either employed/self-employed or other) and education (coded as either primary level or below, secondary level or tertiary level). In order to get a measure of financial status, respondents were also asked how easy it was for their household to make ends meet on a scale of 1 (very easily) to 6 (with great difficulty). Scores for this single-item measure were recoded so that higher scores corresponded to greater ease of making ends meet. Similarly, respondents were asked to rate how good their health was in general on a 5-point single-item scale ranging from 1 (very good) to 5 (very bad). Again, scores were recoded so that higher scores corresponded to better levels of self-rated health.

Social engagement and support. We included a number of different measures of objective social support and engagement, each with a single item. First, a measure of whether the person lived alone was taken. Respondents were also asked how often they had direct face-to-face contact with people living outside their household, with separate questions corresponding to (a) any family members/relatives and (b) any friends/neighbours. Another question asked how often respondents participated in social activities of a club, society or association. For all the above, responses ranged from 1 (every day or almost every day) to 5 (never), which were recoded so that higher scores corresponded to higher levels of engagement.

In addition, respondents were asked hypothetical questions regarding whether they could get support in certain situations. Specifically, they were asked whether they could get help around the house when ill and also whether they could talk to someone if they were feeling a bit depressed. For each of these two questions, respondents were asked where they would get support, from either (a) a member of family/relative, (b) a friend/ 
neighbour, (c) someone else who does not belong to family or relatives or (d) nobody. We recoded responses into a dichotomous variable representing whether a respondent could get support or not $(0=$ no; $1=$ yes $)$, regardless of the source of support.

Social appraisals. Two measures relating to respondents' subjective experience of social support were included, specifically feelings of loneliness and social exclusion. Loneliness was measured using a single item which asked respondents the extent to which they felt lonely over the last 2 weeks, on a scale of 1 (all of the time) to 6 (at no time). Scores were transformed so that higher scores indicated greater levels of loneliness. Social exclusion was determined by the Social Exclusion Index, which focuses on appraisals of social support using five items (e.g. 'I feel left out of society' and 'I feel that the value of what I do is not recognised by others'). Statements were rated on a scale of 1 (strongly agree) to 5 (strongly disagree). Items were summed with higher scores representing higher levels of social exclusion. Reliability was good for this scale (Cronbach's alpha=0.72).

Psychological appraisals. The EQLS included various measures, which we term 'psychological appraisals' (Maguire et al., 2019). We considered these measures to be appraisals due to their focus on an individual's subjective perception of their own abilities and outlook, rather than an objective report of their living circumstances. Specifically, these appraisals comprised measures of (a) optimism ('I am optimistic about my future'), (b) purpose ('I generally feel what I do in life is worthwhile'), (c) autonomy ('I feel I am free to decide how to live my life') and (d) resilience ('I find it difficult to deal with important problems that come up in my life' and 'When things go wrong in my life, it generally takes me a long time to get back to normal'). For all items, respondents rated their agreement on a scale of 1 (strongly agree) to 5 (strongly disagree), with scores recoded so that higher scores related to more positive psychological appraisals. With the exception of resilience (which had two items), all of these variables involved single-item measures.

Analysis. To enable greater ease of interpretation, scores for all continuous variables were standardised from 0 to 100 (see Figure 1 for interpretation). Descriptive statistics were computed for all measures across the different chronic illness groups (i.e. no-limitations, somelimitations and severe-limitations groups). Given that this was a multi-country study, intraclass correlations (ICCs) were then calculated to determine the degree of similarity among respondents in each county across measures and, more importantly, to ascertain whether there was any clustering of results. The results of this analysis informed our decision to employ multilevel regression modelling to explore associations with the predictor variables and psychological well-being (following guidelines from Lee, 2000). We applied the same analytic approach for each of the three illness groups. For purposes of comparison, we also conducted this analysis with the remaining EQLS sample (i.e. those without any chronic illness).

The multilevel analysis initially involved the construction of an intercept-only (null) model to establish the variation in WHO-5 scores across countries (Model 1). This model acted as a reference for comparing the influence of the various explanatory variables in subsequent models across each group (Models 2-5). As can be seen in Figure 1, Model 2 included the addition of sociodemographic and health variables, Model 3 included measures of social engagement and support, Model 4 included social appraisals and Model 5 (the final model) included psychological appraisals. In all models, predictors were included as fixed effects, with the intercept as a random effect. Variance participant coefficients were calculated using the restricted maximum likelihood (RML) technique, and explained variance was estimated following an established procedure (Xu, 2003). Associations with predictor variables and well-being were assessed using two-sided $t$-tests $(p<0.001)$. 


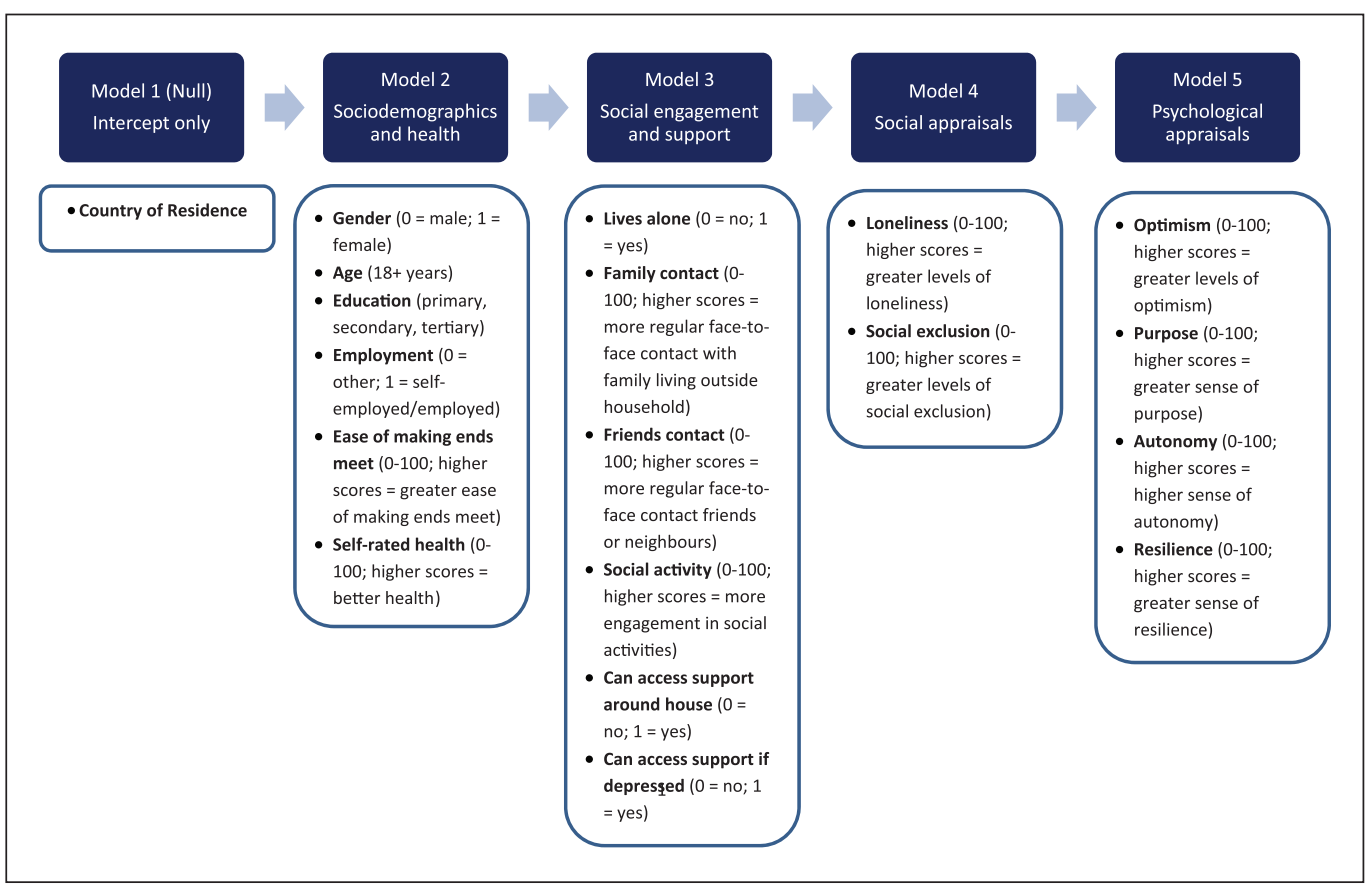

Figure I. Variables included in each of the five multilevel models.

We first ran the analyses using the complete case data and then repeated using multiple imputed data. Here, multiple imputation was applied to replace missing values using an iterative Markov Chain Monte Carlo algorithm. Ten data sets were created, and pooled estimates were produced for all of the five models.

\section{Results}

\section{Descriptive statistics}

A total of 10,577 respondents indicated that they had a chronic illness, representing 29 per cent of the sample. Just over half (52\%) indicated that their illness imposed some limitations on their activities, with a roughly equal amount indicating they were either not limited or severely limited by illness ( $24 \%$ each). The table included in the supplementary material displays detailed information on the number of respondents from each of the 33 participating countries, in addition to the number of respondents in each of the three chronic illness groups. As can be seen here, there was some variation in the percentages of respondents reporting a chronic illness between countries. For example, only 2 per cent of EQLS respondents living in Turkey reported suffering from a condition that severely limited their daily activities, compared to as many as 16 per cent of respondents in Estonia, Latvia and Albania.

The majority of respondents in the EQLS sample were female $(n=20,874 ; 57 \%)$ and ranged in age from 18 to 95 years $(M=50.77$; $\mathrm{SD}=17.1)$. Table 1 displays descriptive statistics for the various chronic illness groups, as well as data for those who did not report an illness Those with severe limitations were more likely to be older $(\mathrm{M}=63.82$ years; $\mathrm{SD}=15.35)$, in worse health $(\mathrm{M}=31.16 ; \mathrm{SD}=21.67)$ and not in employment ( $72 \%$ of group) when compared to the other groups. Well-being decreased for each of the three illness groups, although there was no significant difference between the nolimitations group and the no-illness group. 
Table I. Comparison of chronic illness groups and those without any illness on study measures.

\begin{tabular}{|c|c|c|c|c|c|c|c|c|}
\hline \multirow[b]{2}{*}{ Categorical variables } & \multicolumn{2}{|c|}{$\begin{array}{l}\text { No illness } \\
(N=26,33 \mathrm{I})\end{array}$} & \multicolumn{2}{|c|}{$\begin{array}{l}\text { No limitations } \\
(N=2523)\end{array}$} & \multicolumn{2}{|c|}{$\begin{array}{l}\text { Some } \\
\text { limitations } \\
(N=5370)\end{array}$} & \multicolumn{2}{|c|}{$\begin{array}{l}\text { Severe } \\
\text { limitations } \\
(N=2684)\end{array}$} \\
\hline & $N$ & $\%$ & $N$ & $\%$ & $N$ & $\%$ & $N$ & $\%$ \\
\hline \multicolumn{9}{|l|}{ Gender } \\
\hline Male & 11,917 & 45.3 & 1107 & 43.9 & 2011 & 37.4 & 999 & 37.2 \\
\hline Female & $|4,4| 4$ & 54.7 & 1416 & 56.1 & 3359 & 62.6 & 1685 & 62.8 \\
\hline \multicolumn{9}{|l|}{ Education } \\
\hline Primary or below & 2282 & 8.7 & 272 & 10.8 & 871 & 16.2 & 632 & 23.5 \\
\hline Second level & 16,422 & 62.4 & 1443 & 57.2 & 3406 & 63.4 & 1678 & 62.5 \\
\hline Third level & 7494 & 28.5 & 798 & 31.6 & 1069 & 19.9 & 355 & 13.2 \\
\hline Missing & 133 & 0.5 & 10 & 0.4 & 24 & 0.4 & 19 & 0.7 \\
\hline \multicolumn{9}{|l|}{ Employment status } \\
\hline Employed/self-employed & 14,149 & 53.7 & 1023 & 40.5 & $150 \mid$ & 28.0 & 330 & 12.3 \\
\hline Other & 12,182 & 46.3 & 1500 & 59.5 & 3869 & 72.0 & 2354 & 87.7 \\
\hline \multicolumn{9}{|l|}{ Lives alone } \\
\hline No & 20,763 & 78.9 & 1877 & 74.4 & 3605 & 67.1 & 1642 & 61.2 \\
\hline Yes & 5568 & 21.1 & 646 & 25.6 & 1765 & 32.9 & 1042 & 38.8 \\
\hline \multicolumn{9}{|l|}{ Support around house } \\
\hline No & 652 & 2.5 & 63 & 2.5 & 156 & 2.9 & 125 & 4.7 \\
\hline Yes & 25,558 & 97.1 & 2450 & 97.1 & 5192 & 96.7 & 2547 & 94.9 \\
\hline Missing & $|2|$ & 0.5 & 10 & 0.4 & 22 & 0.4 & 12 & 0.4 \\
\hline \multicolumn{9}{|l|}{ Support when depressed } \\
\hline No & 1004 & 3.8 & 125 & 5.0 & 295 & 5.5 & 213 & 7.9 \\
\hline Yes & 25,021 & 95.0 & 2361 & 93.6 & 5006 & 93.2 & 2428 & 90.5 \\
\hline Missing & 306 & 1.2 & 37 & 1.5 & 69 & 1.3 & 43 & 1.6 \\
\hline Continuous variables & M & SD & M & SD & M & SD & $M$ & SD \\
\hline Age & 47.00 & 16.97 & 56.88 & 16.09 & 59.84 & 15.82 & 63.82 & 15.35 \\
\hline Ease of making ends meet & 55.01 & 25.80 & 58.57 & 26.98 & 47.66 & 27.26 & 37.86 & 29.15 \\
\hline Self-rated health & 76.64 & 18.63 & 63.12 & 18.59 & 49.72 & 18.49 & 31.16 & 21.67 \\
\hline Social activity & 23.08 & 30.16 & 24.71 & 31.50 & 20.58 & 29.67 & $|3.5|$ & 26.23 \\
\hline FTF contact with family & 75.58 & 25.20 & 73.33 & 25.28 & 73.02 & 26.22 & 70.87 & 29.16 \\
\hline FTF contact with friends & 79.81 & 22.14 & 80.70 & 22.10 & 79.44 & 23.17 & 76.06 & 27.05 \\
\hline Social exclusion index & 28.88 & 21.10 & 26.24 & 20.64 & 34.16 & 22.40 & 42.20 & 24.69 \\
\hline Loneliness & $17.5 \mid$ & 24.66 & 15.64 & 25.00 & 24.03 & 28.89 & 36.16 & 35.77 \\
\hline Optimism & 67.51 & 24.38 & 66.30 & 25.53 & 58.39 & 26.42 & 49.52 & 29.70 \\
\hline Purpose & 73.52 & 20.83 & 75.70 & 21.04 & 68.37 & 22.58 & 62.47 & 27.16 \\
\hline Autonomy & 73.16 & 23.45 & 75.42 & 24.28 & 69.03 & 25.39 & 63.68 & 29.85 \\
\hline Resilience & 60.16 & 24.86 & 63.05 & 24.84 & 53.98 & 25.70 & 45.12 & 28.12 \\
\hline WHO-5 & 66.13 & 19.57 & 64.93 & 19.40 & 54.01 & 21.28 & 42.40 & 23.99 \\
\hline
\end{tabular}

FTF: face-to-face; M: mean; SD: standard deviation; WHO-5: World Health Organization Mental Well-Being Index.

Similarly, the no-limitations group did not dif- however, those experiencing limitations had fer from the no-illness group on the psychologi- more negative psychological and social appraiscal and social measures. In comparison, als overall. Observation of effect sizes however 
suggests the majority of differences between groups were small (Cohen's $d<0.5$ ).

\section{ICCs}

An ICC close to zero suggests little variance between countries, whereas larger ICC estimates indicate a greater degree of homogeneity. A list of all ICCs for the study measures is included in the supplementary material. While ICCs were typically low, there were some exceptions, such as the variable 'ease of making ends meet' $(\mathrm{ICC}=0.20)$, levels of social exclusion $(\mathrm{ICC}=0.12)$ and social activity $(\mathrm{ICC}=0.12)$. Where ICCs exceed 10 per cent of the variance of a given outcome, multilevel analysis is recommended (Lee, 2000).

\section{Multilevel regression models}

A series of multilevel regression models were conducted for each of the three chronic illness groups, as well as within the remaining sample. Country of residence was included as a random effect in each model, with other predictors as fixed effects. As a complete case analysis resulted in a loss of 8-14 per cent of cases, with a higher proportion of missing data from the severely limited group, we repeated the analyses using multiple imputed data. Table 2 displays the results. As these were largely similar, we report only the results of the complete case analyses (see supplemental table for results using imputed data).

When compared to the intercept-only models, the addition of sociodemographic and health variables explained 12-18 per cent of variance in well-being across groups, with the largest proportion of variance explained in the severely limited group. Social engagement factors explained an additional 2-3per cent of variance, while social appraisals explained an additional 6-8 per cent of variance. Finally, psychological appraisals explained an additional 5-6per cent of the variance. Overall, the largest proportion of variance was explained in the severely limited chronic illness group (33\%), followed by the group with some limitations (30\%) and the group with no limitations $(27 \%)$. This compares to the remaining EQLS sample without a chronic illness, where only 25 per cent of variance was explained by the model. The supplemental material also includes a figure which illustrates the cumulative variance explained by the various blocks of factors in the complete case analysis for each of the chronic illness groups.

The coefficients of the final multilevel models are shown in Table 3. In all groups, self-rated health emerged as the strongest predictor of wellbeing and was highest in the severely limited group (coefficient $=0.26 ; p<0.001$ ). Ease of making ends meet also emerged as a strong predictor (coefficient range $=0.07-0.10 ; p<0.001$ ), although employment status was only significant in the group without limitations (coefficient $=-4.01 ; p<0.001)$. Specifically, in this group, being employed was associated with a lower level of well-being. In terms of social engagement, living alone and engaging in more social activities was associated with greater wellbeing in the groups limited by illness, while more contact with neighbours or friends was associated with higher well-being for all. Loneliness emerged as a consistent predictor of lower well-being, but was strongest in the group without limitations (coefficient $=-0.14 ; p<0.001$ ). Perceptions of social exclusion were also a significant predictor of lower well-being for the nolimitations and some-limitations groups, but not for the severely limited group. All positive psychological appraisals emerged as significant predictors of well-being across groups, with the exception of autonomy, which did not predict well-being in the severely limited group. The supplementary material also includes a figure which illustrates the relative strength of the different social and psychological appraisals in contributing to the final model. As can be seen here, optimism and resilience were stronger predictors of well-being in the group with severe limitations when compared with the other groups.

\section{Discussion}

This study investigated the experiences of a large sample of Europeans with chronic illness, leading to a number of interesting findings. We 


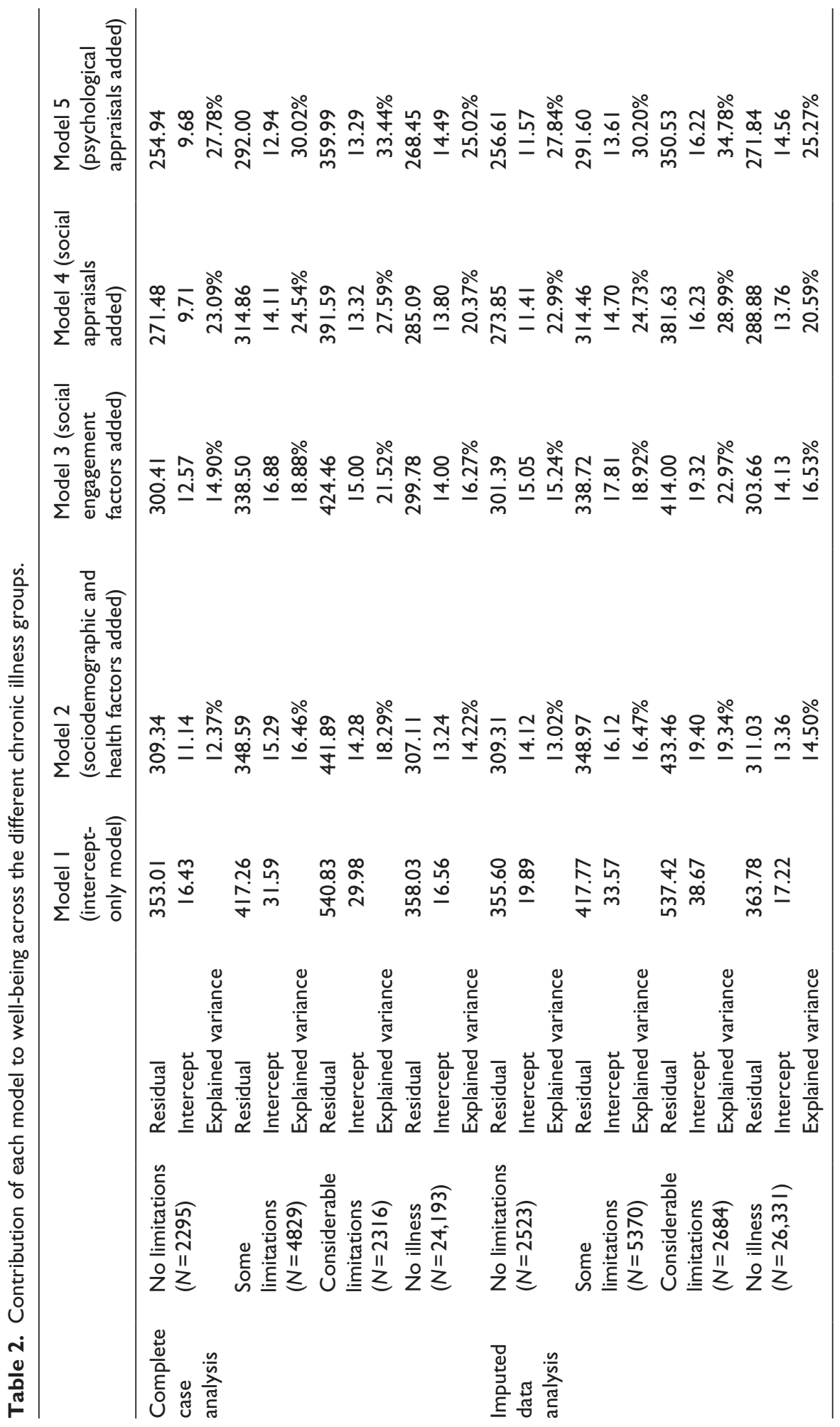




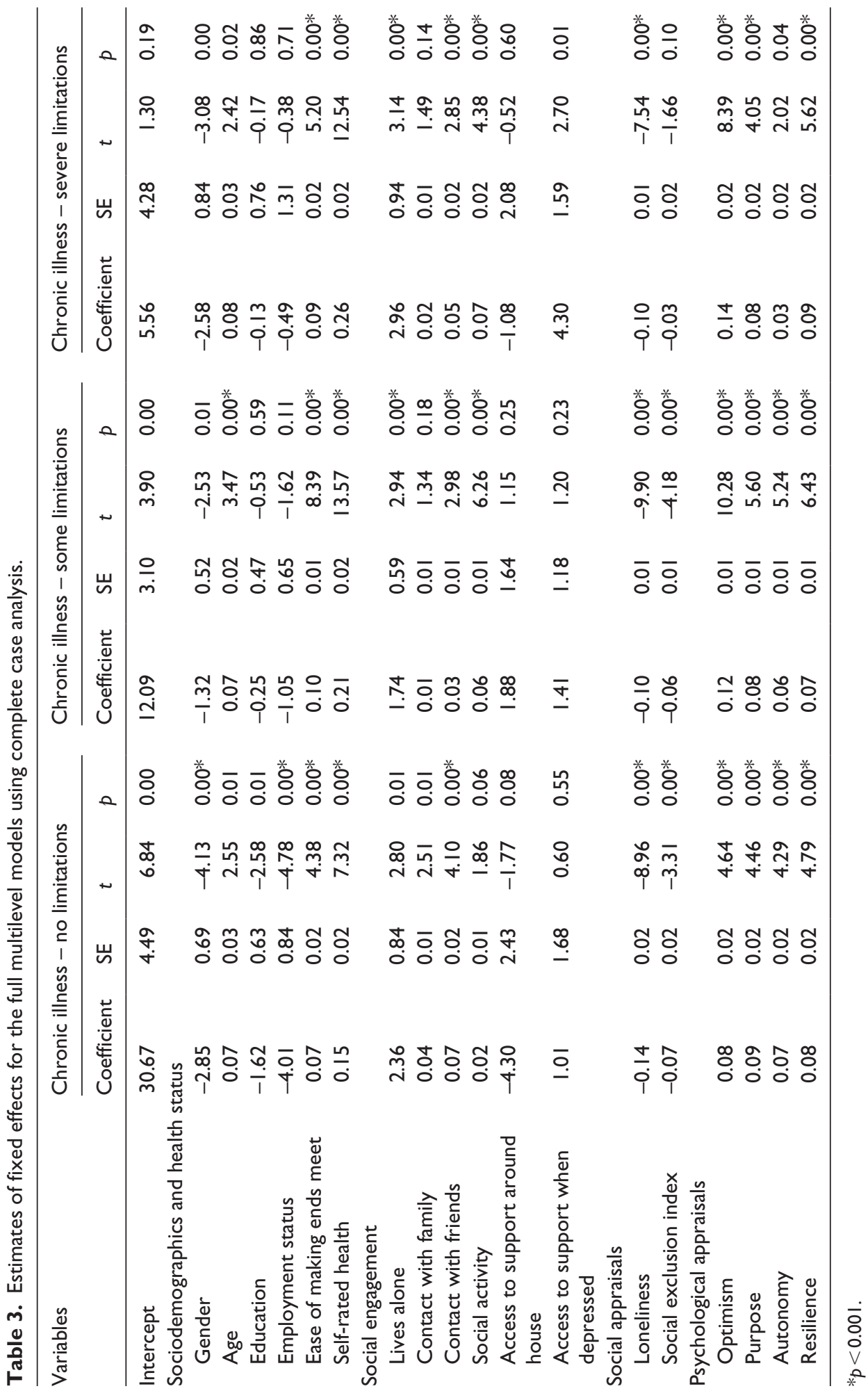


have developed a model of well-being that takes into account various diverse elements of an individual's living circumstances, health and outlook. Overall, this model was good at explaining well-being in all illness groups, but particularly in those individuals who had considerable limitations imposed by their conditions. Our findings highlight the important role that subjective social and psychological appraisals play in determining well-being. Interestingly, we have also shown that these appraisals have differing associations with well-being depending on illness severity.

\section{The role of sociodemographic factors and health status}

In our analysis, we incrementally explored how well-being can be predicted by various factors by first controlling for known sociodemographic associates. Unsurprisingly, health status was the strongest predictor of well-being, becoming even more important with increasing levels of illness severity. Making ends meet was also a strong predictor of well-being, which is line with much literature in the area (Hanly et al., 2018; Taylor et al., 2011). On this point, it is also notable that the ease of making ends meet decreased with illness severity, suggesting that those with limiting illnesses are at particular risk of financial burdens. A potential policy implication for this finding would be to consider the provision of welfare systems on an incremental, as opposed to a dichotomous basis, whereby higher benefits could be considered for those with greater impairments imposed by illness.

Aside from employment status in the nolimitations group, no other sociodemographic factors are associated with well-being, which runs counter to some studies suggesting, for instance, that females are at a risk of lower well-being than males (Bekker and van MensVerhulst, 2007). It is also acknowledged, however, that gender differences in well-being can be contradictory, with some studies suggesting females have higher well-being than males (Lamers et al., 2011).

\section{Objective social engagement versus subjective social appraisals}

We separately examined the role of objective social engagement and support, as distinct from subjective social appraisals, in determining well-being in the different illness groups. A number of interesting findings emerged here. Notably, social engagement factors only contributed to a small amount of variance in wellbeing scores, with the only consistent predictor for all illness groups being contact with neighbours and friends. Specifically, those who engaged in greater contact with neighbours and friends had a higher sense of well-being overall. This reflects other studies which indicate that contact with individuals outside the family is important (Reblin and Uchino, 2008) and that certain characteristics of one's neighbourhood can be critical in enhancing well-being (O’Campo et al., 2009). Counterintuitively, those limited by illness who were living alone had a higher sense of well-being than those who were not, perhaps being indicative of their ability to live independently. Our measure of social activity was also a predictor of well-being in the two groups limited by illness, suggesting that encouraging greater levels of active social participation may be particularly beneficial for those with limitations. This is consistent with previous work (Jang et al., 2004) which revealed that social engagement (specifically social networks and participation in social activities) was more strongly related to life satisfaction in individuals with disease and disability, compared to those with disease and no disability, in an older sample of adults. It is possible, therefore, that a greater value is placed on social participation in those limited by illness.

While the above findings point to the importance of social contact in chronic illness, a much stronger determinant of well-being in all groups was what we have termed 'social appraisals'. Loneliness, in particular, emerged as an important associate of well-being, which is line with a wealth of research in the area (Golden et al., 2009; Lamu and Olsen, 2018). 
Interestingly, we found that loneliness appeared to be more important in determining well-being in the no-limitations group, when compared to the groups limited by their conditions. This may be because those with illness perceive their disability as an objective barrier to social participation and interpret their loneliness in these terms. Social exclusion was also an important predictor of well-being in the no-limitations group, yet did not emerge as a significant predictor in the severe-limitations group. This suggests that, while important, social appraisals are not as central in determining well-being among those with limiting illnesses as compared to those who are not limited by their conditions. However, ways of minimising perceptions of loneliness and social exclusion should be considered in individuals who are diagnosed with a chronic illness that does not impose limitations on their daily activities.

\section{Psychological appraisals}

Finally, our analysis endeavoured to explore the role of psychological appraisals in determining well-being. The most striking observation here was that optimism, while important for all groups, was a far stronger predictor of wellbeing in those with considerable limitations imposed by illness. Indeed, as can be seen in the supplementary figure b, a stepwise trend was observed for the relative importance of optimism in all three groups, with the strongest effect in the severely limited group. While this fits with a number of studies in the area (Karademas, 2006; Scheier et al., 2001), our findings extend upon this work by suggesting that it may be particularly important to foster hope in those who are considerably limited by illness. Although some work (Hurt et al., 2014) has suggested that having an overly optimistic outlook may be detrimental, our findings suggest otherwise in the case of those severely limited by illness.

While not as clear-cut, our analysis suggests that having a good sense of resilience is also important in coping with illness. Resilience is a concept which has been receiving considerable attention in recent years. Our analysis suggests that fostering resilience in those with illness may lead to enhancements in well-being (Cal et al., 2015; Edward, 2013; Gheshlagh et al., 2016). Furthermore, having a sense of purpose was important for all three groups. One interesting finding from our analysis was that perception of autonomy was not a significant determinant for well-being in the group severely limited by illness. While this does seem to be important in those who only have some limitations imposed by illness, our results suggest that other psychological appraisals are more important in those who are severely limited. This runs contrary to the dominant assumption that having a sense of empowerment and autonomy is essential to ensure well-being when coping with illness (Cardol et al., 2002; Naik et al., 2009). It also challenges the assumption from self-determination theory that autonomy is one of the most basic psychological needs (Deci and Ryan, 2008). Our findings may owe to the fact that many individuals who are severely limited by their condition have a greater acceptance of their dependence on others, so that a sense of autonomy is not a necessary influencing factor in well-being. Further research in this area is merited to uncover the exact mechanisms influencing this relationship.

Overall, our analysis points to the important role of positive social and psychological appraisals in maintaining well-being among those with chronic illness. Notably, however, our findings also suggest that the impact of such appraisals can vary depending on individuals' needs.

\section{Limitations}

This study has provided a comprehensive analysis of the various factors that associate with well-being in three categories of individuals with chronic illness from a large European sample. There are, however, a number of limitations which must be acknowledged when considering these findings. First, the study involved a crosssectional design, meaning that we cannot be confident of the direction of the relationships over time. It would be interesting to explore 
how social and psychological appraisals predict later well-being, especially for those whose chronic illnesses are progressive. The study also suffers from a number of methodological limitations. For example, some of the measures used to establish levels of social engagement, psychological appraisals and loneliness were not based on established, standardised questionnaires, but rather on single-item measures, which may restrict their reliability.

It should also be acknowledged that we used only one measure of well-being in our study. While the WHO-5 is a widely employed scale which has been validated in many settings (Topp et al., 2015), there are many other definitions and measures of psychological well-being in existence (McDowell, 2010) such as the Ryff scale of psychological well-being (Ryff and Singer, 1998). Interestingly, this particular scale includes some items which measure what we have termed 'psychological appraisals' (e.g. autonomy and sense of purpose are also components of Ryff's theory). Thus, according to this definition, such appraisals form part of one's overall subjective well-being, rather than being simply related to it. In our analysis, we considered appraisals as separate to global psychological well-being (as measured by the WHO-5 index); the variations in theoretical approaches to understanding well-being may call this assumption into question.

Finally, other than restrictions on daily activities and self-rated health status, no other information was obtained on the nature of respondents' condition. It would be expected, for example, that conditions such as rheumatoid arthritis or certain forms of cancer may lead to considerable pain which may impact on one's quality of life, regardless of functional limitations. The stage or severity of one's illness at any given time may also have an effect. For example, in relapsing remitting multiple sclerosis, well-being may be differently impacted depending on whether one is experiencing a relapse or is in remission. It is likely that having access to such detailed information would give further insight into how various factors influence well-being in people with illness.

\section{Conclusion and implications}

Merely having a chronic illness does not mean that well-being should suffer. As evidence of this, a sizable proportion of our sample had conditions that were not considered limiting. Our findings suggest that in order to identify supports for people with illness, it is important to first consider the extent to which they are limited by their conditions. While loneliness and social engagement are crucial determinants of wellbeing, psychological appraisals appear to be more important in those who are limited in their activities when compared to those who are not. Fostering a sense of optimism and resilience may be particularly important when intervening to increase well-being in those limited by illness. Conversely, maintaining a sense of autonomy may be less important for this group. These findings open up many new directions regarding potential means in which well-being can be maintained. In particular, policymakers may wish to consider how modifiable social and psychological factors may be targeted to enhance well-being in those coping with illness.

\section{Declaration of Conflicting Interest}

The author(s) declared no potential conflicts of interest with respect to the research, authorship and/or publication of this article.

\section{Funding}

The author(s) received no financial support for the research, authorship and/or publication of this article.

\section{ORCID iD}

Rebecca Maguire (iD https://orcid.org/0000-0002 $-0368-4626$

\section{Supplemental material}

Supplemental material for this article is available online.

\section{References}

Bekker MH and van Mens-Verhulst J (2007) Anxiety disorders: Sex differences in prevalence, degree, 
and background, but gender-neutral treatment. Gender Medicine 4: S178-S193.

Boyle PA, Barnes LL, Buchman AS, et al. (2009) Purpose in life is associated with mortality among community-dwelling older persons. Psychosomatic Medicine 71(5): 574-579.

Brennan P, Perola M, van Ommen G-J, et al. (2017) Chronic disease research in Europe and the need for integrated population cohorts. European Journal of Epidemiology 32(9): 741-749.

Busse R and Blümel M (2010) Tackling Chronic Disease in Europe: Strategies, Interventions and Challenges. Copenhagen: WHO Regional Office Europe.

Cal SF, de Sá LR, Glustak ME, et al. (2015) Resilience in chronic diseases: A systematic review. Cogent Psychology 2(1): 1024928.

Cardol M, Jong BD and Ward CD (2002) On autonomy and participation in rehabilitation. Disability and Rehabilitation 24(18): 970-974.

Chakhssi F, Kraiss JT, Sommers-Spijkerman M, et al. (2018) The effect of positive psychology interventions on well-being and distress in clinical samples with psychiatric or somatic disorders: A systematic review and meta-analysis. BMC Psychiatry 18(1): 211.

De Ridder D, Geenen R, Kuijer R, et al. (2008) Psychological adjustment to chronic disease. The Lancet 372(9634): 246-255.

Deci EL and Ryan RM (2008) Self-determination theory: A macrotheory of human motivation, development, and health. Canadian Psychology/ Psychologie Canadienne 49(3): 182-185.

Delle Fave A, Bassi M, Allegri B, et al. (2017) Beyond disease: Happiness, goals, and meanings among persons with multiple sclerosis and their caregivers. Frontiers in Psychology 8: 2216.

Dezutter J, Luyckx K and Wachholtz A (2015) Meaning in life in chronic pain patients over time: Associations with pain experience and psychological well-being. Journal of Behavioral Medicine 38(2): 384-396.

Diener E and Chan MY (2011) Happy people live longer: Subjective well-being contributes to health and longevity. Applied Psychology 3(1): $1-43$.

DuBois CM, Lopez OV, Beale EE, et al. (2015) Relationships between positive psychological constructs and health outcomes in patients with cardiovascular disease: A systematic review. International Journal of Cardiology 195: 265-280.
Edward K (2013) Chronic illness and wellbeing: Using nursing practice to foster resilience as resistance. British Journal of Nursing 22(13): 741-746.

Eiser JR, Riazi A, Eiser C, et al. (2001) Predictors of psychological well-being in types 1 and 2 diabetes. Psychology \& Health 16(1): 99-110.

Eurofound (2018) European Quality of Life Survey Integrated Data File, 2003-2016 (Data collection, SN: 7348, 3rd edn). Available at: https:// beta.ukdataservice.ac.uk/datacatalogue/studies/ study?id $=7348$

Geerling B, Kraiss J, Kelders S, et al. (2019) The effect of positive psychology interventions on well-being and psychopathology in patients with severe mental illness; A meta-analysis. Bipolar Disorders 21(S1): 126-127.

Gheshlagh RG, Sayehmiri K, Ebadi A, et al. (2016) Resilience of patients with chronic physical diseases: A systematic review and meta-analysis. Iranian Red Crescent Medical Journal 18(7): e38562.

Golden J, Conroy RM, Bruce I, et al. (2009) Loneliness, social support networks, mood and wellbeing in community-dwelling elderly. International Journal of Geriatric Psychiatry 24(7): 694-700.

Hanly P, Maguire R, Ceilleachair AO, et al. (2018) Financial hardship associated with colorectal cancer survivorship: The role of asset depletion and debt accumulation. Psycho-Oncology 27(9): 2165-2171.

Hurt CS, Burn DJ, Hindle J, et al. (2014) Thinking positively about chronic illness: An exploration of optimism, illness perceptions and well-being in patients with Parkinson's disease. British Journal of Health Psychology 19(2): 363-379.

Jang Y, Mortimer JA, Haley WE, et al. (2004) The role of social engagement in life satisfaction: Its significance among older individuals with disease and disability. Journal of Applied Gerontology 23(3): 266-278.

Karademas EC (2006) Self-efficacy, social support and well-being: The mediating role of optimism. Personality and Individual Differences 40(6): 1281-1290.

Kim JH, McMahon BT, Hawley C, et al. (2016) Psychosocial adaptation to chronic illness and disability: A virtue based model. Journal of Occupational Rehabilitation 26(1): 45-55.

Lamers SM, Westerhof GJ, Bohlmeijer ET, et al. (2011) Evaluating the psychometric properties of the 
mental health continuum-short form (MHC-SF). Journal of Clinical Psychology 67(1): 99-110.

Lamu AN and Olsen JA (2018) Yes, health is important, but as much for its importance via social life: The direct and indirect effects of health on subjective well-being in chronically ill individuals. Health Economics 27(1): 209-222.

Lee VE (2000) Using hierarchical linear modeling to study social contexts: The case of school effects. Educational Psychologist 35(2): 125-141.

McDowell I (2010) Measures of self-perceived wellbeing. Journal of Psychosomatic Research 69(1): 69-79.

Maguire R, Hanly P and Maguire P (2019) Beyond care burden: Associations between positive psychological appraisals and well-being among informal caregivers in Europe. Quality of Life Research 28: 2135-2146.

Maguire R, Hanly P, Drummond FJ, et al. (2018) Expecting the worst? The relationship between retrospective and prospective appraisals of illness on quality of life in prostate cancer survivors. Psycho-oncology 27(4): 1237-1243.

Naik AD, Dyer CB, Kunik ME, et al. (2009) Patient autonomy for the management of chronic conditions: A two-component re-conceptualization. The American Journal of Bioethics 9(2): 23-30.
O'Campo P, Salmon C and Burke J (2009) Neighbourhoods and mental well-being: What are the pathways? Health \& Place 15(1): 56-68.

Reblin M and Uchino BN (2008) Social and emotional support and its implication for health. Current Opinion in Psychiatry 21(2): 201-205.

Ryff CD and Singer B (1996) Psychological wellbeing: Meaning, measurement, and implications for psychotherapy research. Psychotherapy and Psychosomatics 65(1): 14-23.

Ryff CD and Singer B (1998) The contours of positive human health. Psychological Inquiry 9(1): $1-28$.

Scheier MF, Carver CS and Bridges MW (2001) Optimism, pessimism, and psychological wellbeing. Optimism and Pessimism 1: 189-216.

Steptoe A, Deaton A and Stone AA (2015) Subjective wellbeing, health, and ageing. The Lancet 385(9968): 640-648.

Taylor MP, Jenkins SP and Sacker A (2011) Financial capability and psychological health. Journal of Economic Psychology 32(5): 710-723.

Topp C, Ostergaard S, Sondergaard S, et al. (2015) The WHO-5 Well-Being Index: A systematic review of the literature. Psychotherapy and Psychosomatics 84(3): 167-176.

$\mathrm{Xu}$ R (2003) Measuring explained variation in linear mixed effects model. Statistics in Medicine 22(22): 3527-3541. 